\title{
THE ROLE OF SPLENECTOMY AND DISTAL PANCREATECTOMY AS CYTOREDUCTIVE PROCEDURES IN OVARIAN CANCER PATIENTS WITH UPPER ABDOMINAL INVOLVEMENT
}

\author{
Yavor Kornovski ${ }^{1,2}$, Elis Ismail ${ }^{1,2}$ \\ ${ }^{1}$ Department of Obstetrics and Gynecology, Medical University of Varna, \\ ${ }^{2}$ Gynecological Clinic, MHAT St. Anna,Varna
}

\begin{abstract}
Ovarian cancer has the highest mortality rate of all gynecologic cancers as $62 \%$ of cases are diagnosed at an advanced stage. The main concern in the surgical management of ovarian cancer is to achieve optimal cytoreductive surgery. Effective cytoreductive surgery at the time of initial therapy has been identified as the most important prognostic factor in the management of advanced ovarian cancer. This aggressive approach can cause unusual surgical management modalities including diaphragm stripping/resection, bowel resection, hepatic resection and splenectomy. Thus, splenectomy occasionally happens to be part of the ovarian cancer surgery.

The indications and technique of splenectomy and distal pancreatectomy as cytoreductive procedures in ovarian cancer patients were described. The authors have presented the indications and techniques of splenectomy and distal pancreatectomy as cytoreductive procedures in ovarian cancer patients. Intraoperative and postoperative complications were reported in this study.
\end{abstract}

Keywords: ovarian cancer, splenectomy

\section{INTRODUCTION}

Splenectomy with or without distal pancreatectomy is part of cytoreductive surgery, which is used in the treatment of advanced and recurrent ovarian cancer in order to achieve complete cytoreduction.

The indications for splenectomy are:

\footnotetext{
Address for correspondence:

Yavor Kornovski

Gynecological Clinic

MHAT St. Anna, Varna

100A Tzar Osvoboditel Blvd.

Varna

e-mail:ykornovski@abv.bg
}

Received: October 20, 2016

Accepted: December 27, 2016 a) direct involvement of metastatic omentum (omental cake) or peritoneal metastasis (in left hemidiaphragm) - in more than $80 \%$ of the cases with advanced cancer or recurrent ovarian cancer

b) solitary or parenchymal splenic metastases (rarely)

c) Iatrogenic injury of the spleen during mobilization of the surrounding tissues.

The spleen is involved by metastases in part of the omentum after incomplete omentectomy (infracolic) (1). Manci (2006) reported 24 patients with involvement of the spleen: the hilum was affected in 12 and 12 had metastases on the surface or parenchyma (1). Splenectomy did not lead to the increase of early postoperative morbidity and according to the authors this procedure is safe as part of cyto- 
reductive surgery. Otrock (2006) reported 17 cases with solitary metastases in the spleen which occurred between 2 and 10 years after the primary treatment, mostly hematogenously (2). According to Farrias E (1993) solitary spleen metastases were diagnosed by CA-125 elevation and PET/CT. Furukawa (2007) reported excellent survival in cases of solitary metastases when surgery and subsequent chemotherapy (CT) (Platinum) were applied $(3,4)$. Intraoperative splenic injures are the third reason, mainly in emergency splenectomy. They usually occur after pulling anatomical structures (mostly omentum or flexura lienalis of colon transversum), which form dense adhesions with the spleen. Fig. 1, 2 and 3 demonstrate the main indications for splenectomy.

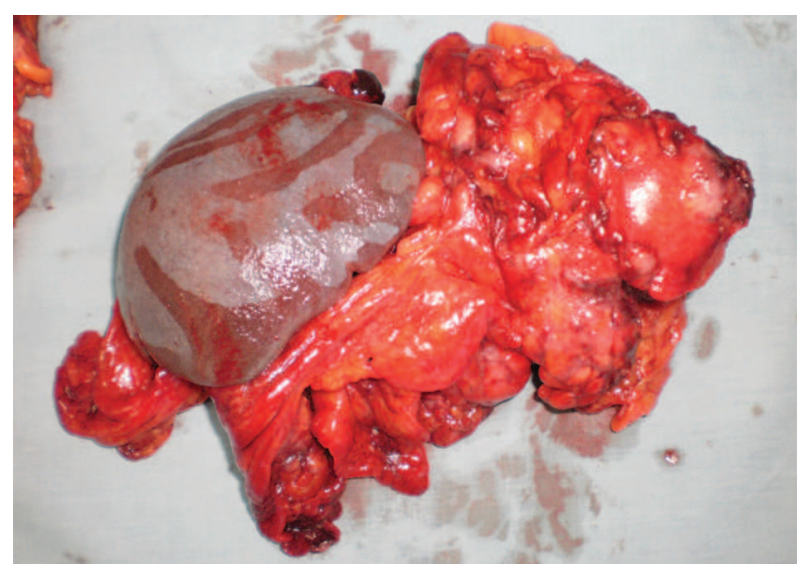

Fig. 1. Direct involvement of metastatic omentum (Y. Kornovski and co-authors)

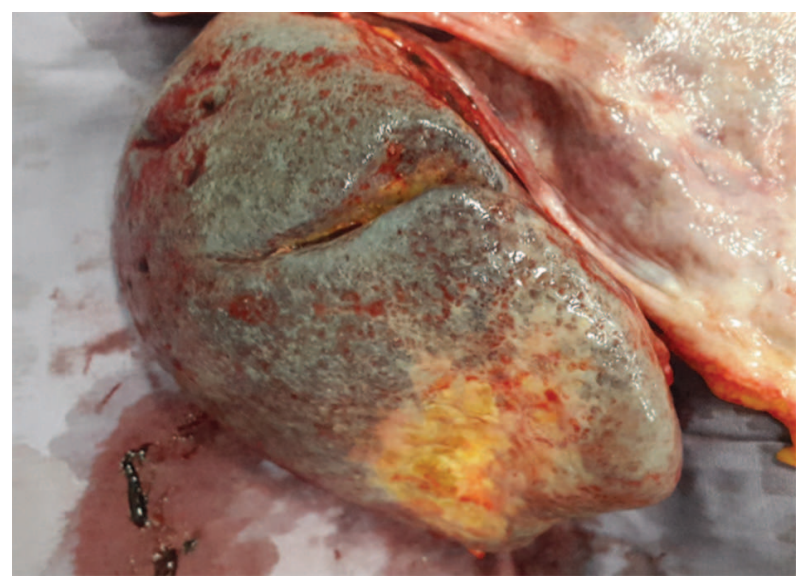

Fig. 2. Solitary metastases (Y. Kornovski and co-authors)

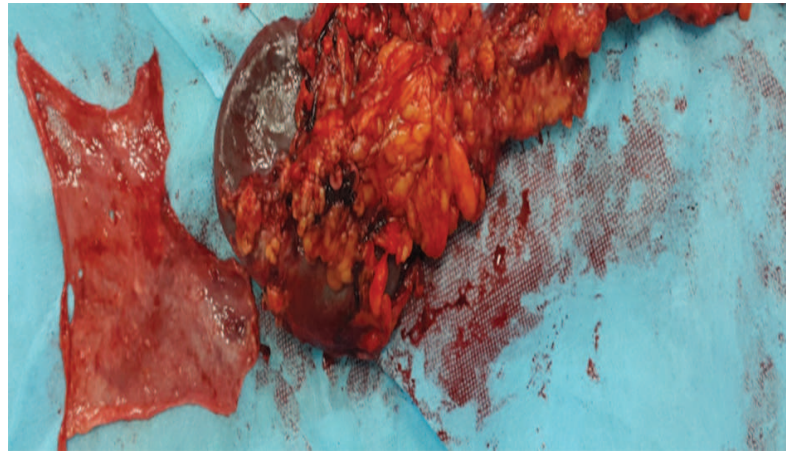

Fig. 3. Hilar splenic tumor infiltration (Y. Kornovski and co-authors)

\section{SURGICAL TECHNIQUE}

There are two main steps when performing splenectomy:

1. The mobilization of the spleno-pancreatic complex. It consists of liberation (ligation and transaction) of the gastrocolic and gastrolienal ligament, which are part of the great omentum and the splenocolic and phrenocolic ligament, as well. Mobilization and supracolic omentectomy are performed simultaneously (Fig. 4).

2. Separating the spleen from the pancreatic tail or its transection (distal pancreatectomy) and ligation of lienal artery and vein.

Two approaches are used: ventral (the separation is performed from the visceral surface of the spleen) and dorsal - from its parietal surface (Fig. 5, Fig. 6).

During the ventral approach there is a risk of damage of the pancreas and conditions for postoperative pancreatic fistula and this approach should be performed only in cases with hilar pancreatic metastases which involve the pancreatic tail and require distal pancreatectomy with splenectomy.

In all other conditions without involvement of the pancreatic tail, the dorsal approach should be performed because it is safe and prevents pancreatic fistulas. The dorsal approach has an anatomic bloodless approach between the tail of pancreas and the lienal artery and vein (Fig. 7, Fig. 8).

After the presenting the lienal vessels they are clamped and the spleen is removed with the metastases. 
Yavor Kornovski, Elis Ismail

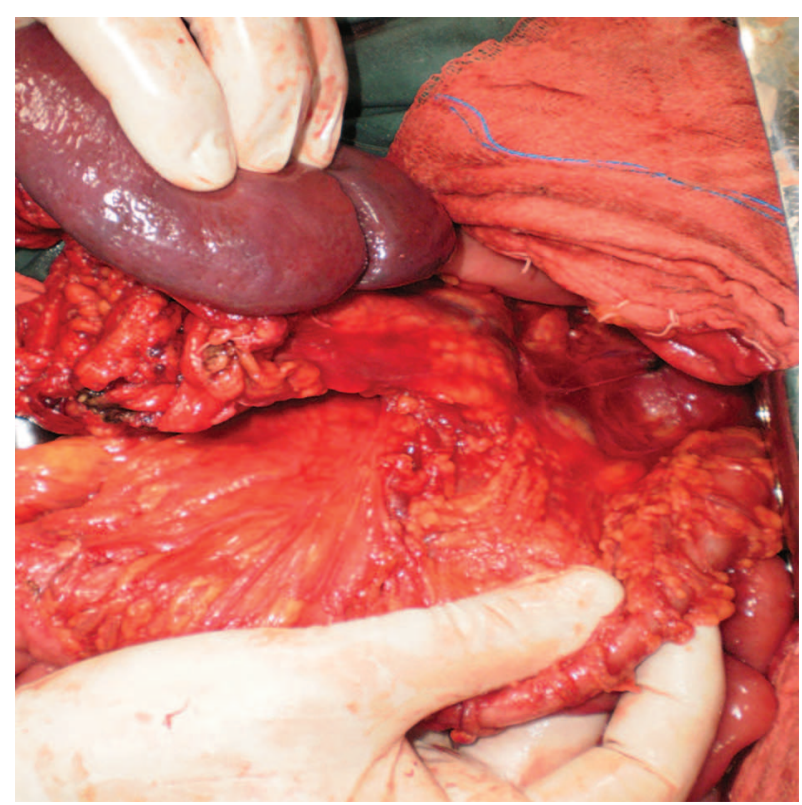

Fig. 4. Spleen and pancreas after mobilization (Y. Kornovski and co-authors)

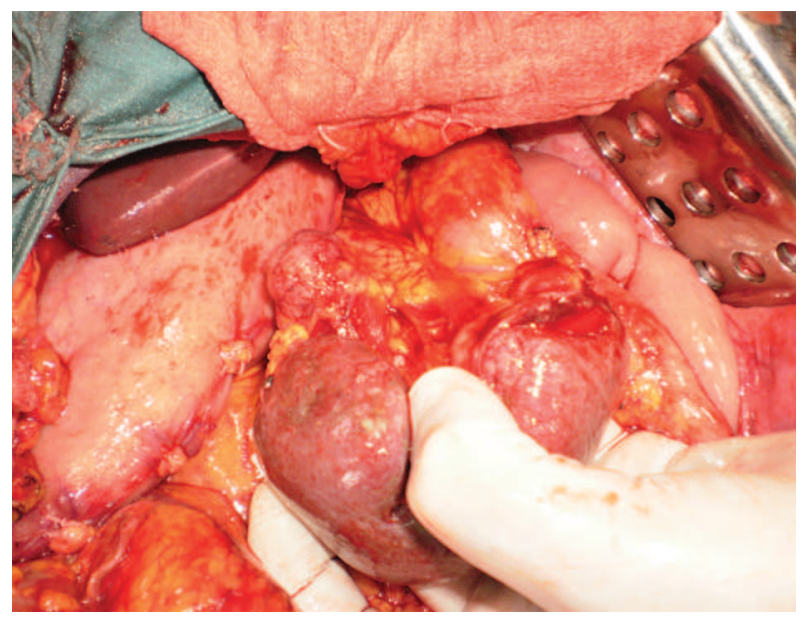

Fig. 5. Ventral approach (Y. Kornovski and co-authors)

The clamping is carried out at distance of the hilum of the spleen where there are many lienal branches that may cause unnecessary bleeding.

\section{PATIENTS' CHARACTERISTICS}

Between November, 2014 and February, 2015 (3 months) in the Gynecological Clinic of MHAT St.Anna, Varna during cytoreductive surgeries - 9 splenectomies and 1 distal pancreatectomy were performed. The distribution by stage was: 8 - IIIC FIGO stage and 1 - IV stage. The types of cytoreductive surgeries are: interval (after neoadjuvant chemotherapy) - in 3 patients, secondary - in 2 patients, recur-

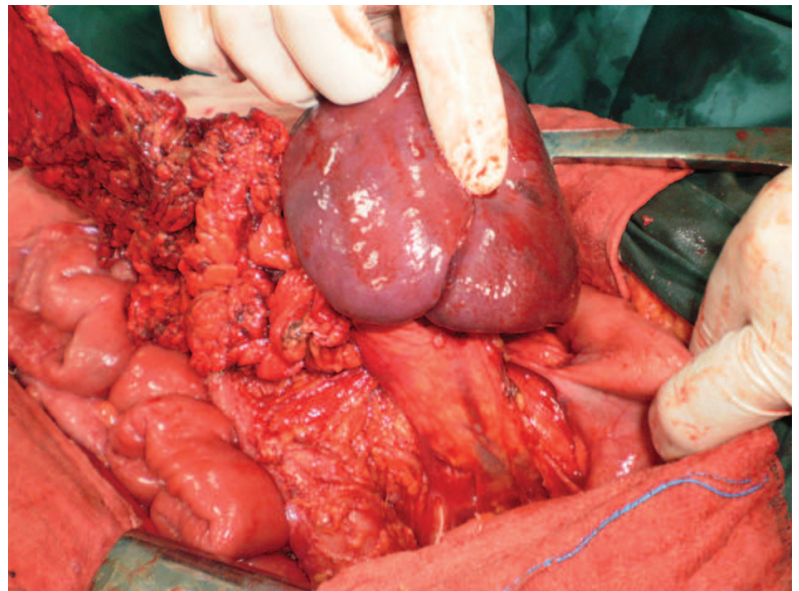

Fig. 6. Dorsal approach (Y. Kornovski and co-authors)

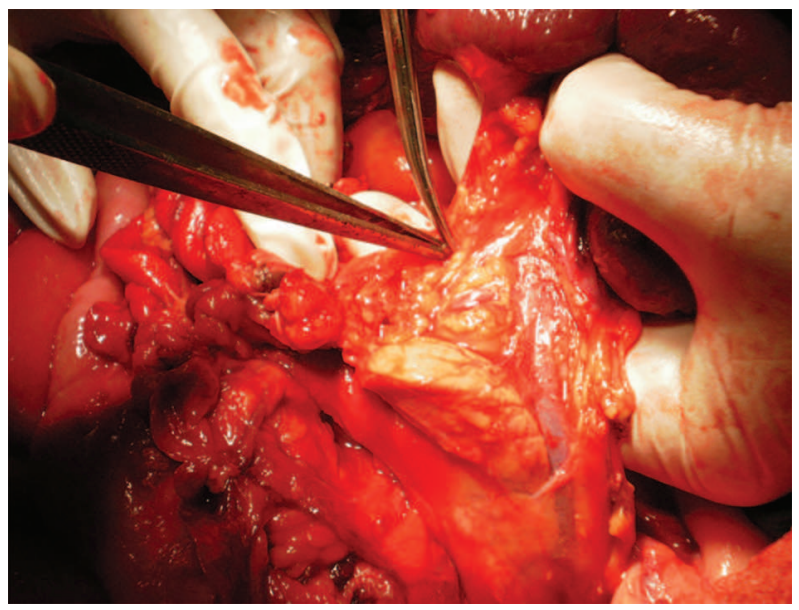

Fig. 7. Sparing the pancreatic tail

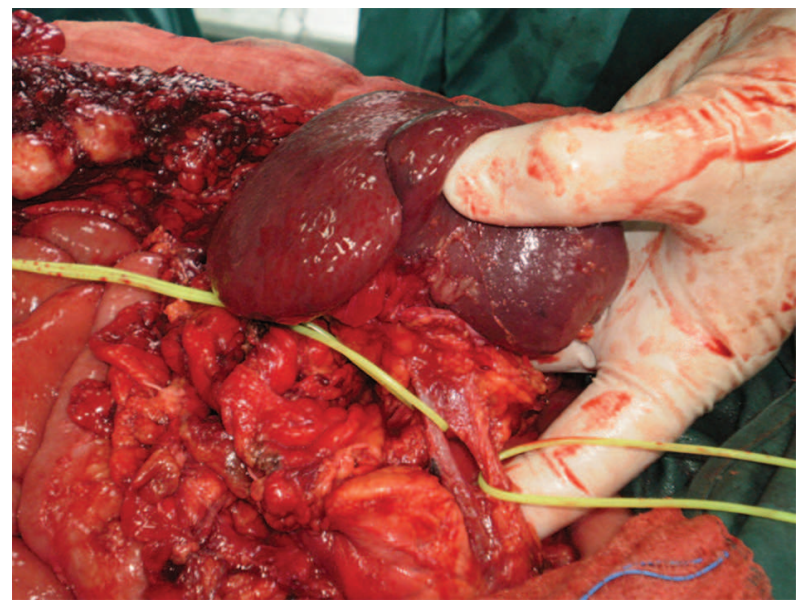

Fig. 8. Presenting lienal artery and vein (Y. Kornovski and co-authors)

rent ovarian cancer - 1 patient and during primary surgery - in 3 patients. According to the extent of sur- 
The Role of Splenectomy and Distal Pancreatectomy as Cytoreductive Procedures in Ovarian Cancer Patients with Upper Abdominal ...

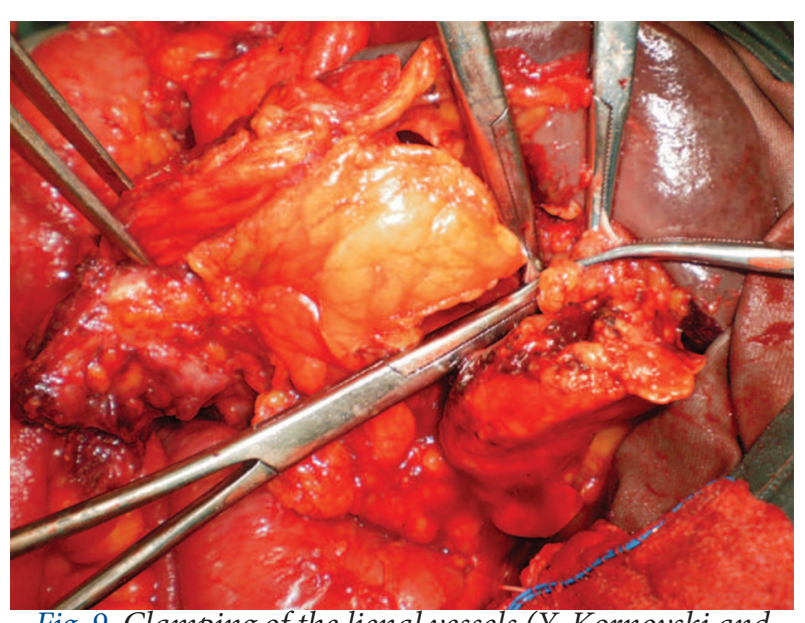

Fig. 9. Clamping of the lienal vessels (Y. Kornovski and co-authors)

gery: splenectomy and supracolic omentectomy was performed in 8 patients; in 1- splenectomy and distal pancreatectomy; in 2- stripping of the left diaphragm. Intraoperative complications occurred in 1 patient - lesion of the left hemidiaphragm which was sutured with continuous stitch. Postoperative complications were observed in two patients: 1 - excessive hypoproteinaemia and mild acute renal failure and 1 - mesenteric thrombosis (on the 6th postoperative day).

The hospital stay was 8 days, intraoperative blood loss - minimal and the time of splenectomy- 45 min (mobilization - 30min., spleen - pancreatic separation or pancreatic tail resection $-15 \mathrm{~min}$.)

Patients' outcome - 1 patient died after mesenteric thrombosis, 1- patients with chylous ascites after distal pancreatectomy, other patients were alive until June 2016.

\section{DISCUSSION}

Eisenkop SM, (2006) compares 47 cases with splenectomy and 307 cases without splenectomy at the time of primary cytoreductive surgery.

Splenectomy was associated with more advanced stage and more extensive surgery. According to the author splenectomy leads to better survival rate when a complete cytoreduction was achieved; does not increase postoperative complications and it is associated with longer operative time. The procedure does not change blood loss and hospital stay (4).
Magtibay (2006) reported 66 cases of splenectomy during primary cytoreduction without complications (5).

The resection of a part of the pancreatic tail with splenectomy increases the risk of complications, mostly pancreatic fistulas.

Yildirim Y. (2005) performed resection of the tail of the pancreas in patients with pancreatic metastases in $6 \%$ of the cases with advanced ovarian cancer, and the procedure led to complete cytoreduction and acceptable morbidity (6).

Kehoe SM (2009) presented 17 cases with distal pancreatectomy and the rate of complication was $24 \%$ (pancreatic leakage) (7).

\section{CONCLUSIONS}

1. The indication for splenectomy, as a part of cytoreductive surgery, is primary advanced and recurrent ovarian cancer.

2. Splenectomy (with or without distal pancreatectomy) increases the operative time, does not change the blood loss and the postoperative morbidity.

3. Splenectomy is associated with better survival rate when complete cytoreduction is achieved.

\section{REFERENCES}

1. Manci $\mathrm{N}$ et al. .Splenectomy during secondary cytoreduction for ovarian cancer disease recurrence: surgical and survival data.Ann Surg Oncol 2006 Dec;13(12):1717-23

2. Otrock ZK et al. Laparoscopic splenectomy for isolated parenchymal splenic metastasis of ovarian cancer.Int J Gynecol Cancer, 2006 Sep-Oct;16(5):1933-5.

3. Farrias-Eisner $\mathrm{R}$ et al. Solitary recurrent metastasis of epithelial ovarian cancer in the spleen. Gynecol Oncol, 1993 Mar;48(3):338-41.

4. Eisenkop SM et al. Splenectomy in the context of primary cytoreductive operations for advanced epithelial ovarian cancer. Gynecol Oncol 2006; 100:344-348

5. Magtibay PM et al. Splenectomy as part of cytoreductive surgery in ovarian cancer. Gynecol Oncol, 2006 Aug;102(2):369-74. Epub 2006 Apr 24.

6. Yildirim Y, Sanci M. The feasibility and morbidity of distal pancreatectomy in extensive cytoreduc- 
tive surgery for advanced epithelial ovarian cancer. Arch Gynecol Obstet 2005;272:31-34

7. Kehoe $\mathrm{SM}$ et al. Incidence and management of pancreatic leaks after splenectomy with distal pancreatectomy performed during primary cytoreductive surgery for advanced ovarian, peritoneal and fallopian tube cancer. Gynecol Oncol ,2009 Mar;112(3):496-500. 